\title{
ON TWO PROBLEMS OF MOSTERT AND SHIELDS
}

\section{J. H. URSELL}

Introduction. A topological semigroup $S$ is a topological space $S$ such that the set $S$ has a binary composition which is associative and continuous with respect to the topology. In [2], P. S. Mostert and A. L. Shields gave a list of problems in topological semigroups. In this and subsequent notes we give partial answers to several of these problems and to others suggested to the author by R. P. Hunter. We give here the answers to problems $\mathrm{P} 2$ and $\mathrm{P} 6$ of [2], which are:

P2. Let $S$ be a locally-compact, connected, Hausdorff topological semigroup with zero and identity. Is there is a compact, connected subsemigroup $M$ containing the zero and identity?

P6. Let $S$ be a compact, connected, Hausdorff topological semigroup with identity and which is not a group. Does $S$ admit a continuous, nontrivial homomorphism on to some $(I)$-semigroup?

In each case we give a counter-example which answers these questions in the negative.

The author wishes to state here his gratitude to his supervisor, M. F. Atiyah, for discussions, and to R. P. Hunter for communications by letter, which have enabled him to present both the theorems in this paper in a far more elegant manner than would otherwise have been the case.

1. Counterexample to P2. Let $X$ be the subspace of the Euclidean plane $R^{2}$ given by

$$
0<x \leqq 1, \quad 0 \leqq y \leqq 1 \text { and } y<\frac{1}{2} \text { if } x=0 .
$$

Then $X$ is locally-compact, Hausdorff and arcwise-connected. We define a multiplication on $X$ as follows:

$$
(x, y) \cdot\left(x^{\prime}, y^{\prime}\right)=\left(x x^{\prime} \operatorname{Max}\left(y, y^{\prime}\right), \operatorname{Min}\left(y, y^{\prime}\right)\right) \text {. }
$$

This is well defined. For each coordinate of the product lies between 0 and 1 and if $\operatorname{Min}\left(y, y^{\prime}\right) \geqq \frac{1}{2}$, then $x, x^{\prime}>0$ and hence $x x^{\prime} \operatorname{Max}\left(y, y^{\prime}\right)>0$. We observe that $(0,0)$ is the zero of $X,(1,1)$ the unit of $X$, and that the multiplication is commutative.

We now prove associativity. That of the second coordinate of a triple product is immediate. Again, by the commutativity,

$$
\left(x x^{\prime}\right) x^{\prime \prime} .=x^{\prime \prime}\left(x^{\prime} x\right), \quad x\left(x^{\prime} x^{\prime \prime}\right)=\left(x^{\prime \prime} x^{\prime}\right) x .
$$

Hence, in order to prove associativity for the first coordinate of a

Received by the editors March 16, 1962 and, in revised form, May 28, 1962. 
triple product, we can without loss of generality assume $y \geqq y^{\prime \prime}$. We now need a lemma on ordered sets.

LEMMA 1. Let $T$ be a set totally ordered by $\leqq$, and write $\operatorname{Max}(a, b)$ $=a \cup b, \operatorname{Min}(a, b)=a \cap b$, all $a, b \in T$. Then for any $a, b, c \in T$, the two unordered pairs $\{a \cap b,(a \cup b) \cap c\},\{a \cap(b \cup c), b \cap c\}$ coincide.

Proof. If $b \geqq a$ or $b \geqq c$, then

$$
\begin{aligned}
& a \cap b=(a \cap b) \cup(a \cap c) \equiv a \cap(b \cup c), \\
& c \cap b=(c \cap b) \cup(a \cap c) \equiv(a \cup b) \cap c .
\end{aligned}
$$

If $b \leqq a$ and $b \leqq c$, then

$$
\begin{aligned}
a \cap b & =c \cap b, \\
a \cap(b \cup c) & \equiv(a \cap b) \cup(a \cap c)=(c \cap b) \cup(a \cap c) \equiv(a \cup b) \cap c .
\end{aligned}
$$

This lemma proves the associativity of $X$. The continuity of the multiplication on $X$ follows because Min, Max and product are all continuous on the reals.

Now for any $(x, y) \in X,(x, y)^{n}=\left(x^{n} y^{n-1}, y\right)$. Hence if $(x, y) \neq(1,1)$,

$$
(x, y)^{n} \rightarrow(0, y) \text { as } n \rightarrow \infty \text {. }
$$

But if $y \geqq \frac{1}{2},(0, y) \notin X$. Thus any subsemigroup containing such an element cannot be compact. If a subsemigroup does not contain such an element and yet contains 0 and 1 , it is not connected.

Hence we have the following

THEOREM 1. There exists an arcwise-connected, locally-connected, locally-compact, Hausdorff topological semigroup with zero and identity such that no compact connected subsemigroup contains both zero and identity.

This includes the solution of problem P2 of Mostert and Shields.

2. Counterexample to P6.

Definition. An $(I)$-semigroup is a topological semigroup on the unit interval such that one endpoint is the zero and the other endpoint is the unit.

Such semigroups have been completely classified by Mostert and Shields in [2], building on results of Faucett in [1].

Let $I=[0,1]$ with multiplication $s \cdot s^{\prime}=\operatorname{Min}\left(s, s^{\prime}\right)$. Let $J=\left[\frac{1}{2}, 1\right]$ with multiplication $t \cdot t^{\prime}=\operatorname{Max}\left(\frac{1}{2}, t t^{\prime}\right)$. Set $S=I \times J$ with coordinatewise multiplication. Notice that

$$
M=[0,1] \times\left\{\frac{1}{2}\right\} \cup\{0\} \times\left[\frac{1}{2}, 1\right]
$$


is an ideal of $S$, that is, $S M \subseteq M, M S \subseteq M$. Define $X=S / M$, the Rees quotient obtained by identifying $M$ to a point, denoted $z \in X$. Thus $X$ is a commutative semigroup with unit $(1,1)$ and zero $z$. Having a zero, it cannot be a group. $X$ is arcwise-connected and compact, being the quotient space of such a space. $X$ is Hausdorff because $S$ is a regular space and $M$ is closed in $S$. The multiplication in $X$ is continuous because that in $S$ is so.

We observe that there is an arc of idempotents from 0 to 1 in $X$, namely $[0,1] \times\{1\}$. All other points of $X$ are strictly nilpotent, that is, for each such $x \in X$, there is an integer $N(x)>1$ such that $x^{N(x)}$ $=0 \in X$.

Let $A$ be any topological semigroup on the unit interval. Suppose there exists a continuous homomorphism $f: X \rightarrow A$. Let $f(1)=a_{1}$, $f(0)=a_{0}, a_{1}, a_{0} \in A$. Since $f$ is a homomorphism, $a_{0}$ and $a_{1}$ are idempotents. Suppose $a_{1}=a_{0}$. Let $x \in X$; then

$$
f(x)=f(x \cdot 1)=f(x) \cdot f(1)=f(x) \cdot f(0)=f(x \cdot 0)=f(0)=a_{0} .
$$

Thus $f(X)=\left\{a_{0}\right\}$, and $f$ is the trivial map onto an idempotent singleton.

If $a_{0} \neq a_{1}$, consider $a_{0}<a_{1}$. Choose $b \in A, a_{0}<b<a_{1}$. Then $b=f(y)$ $=f\left(y^{\prime}\right)$ where $y$ is idempotent and $y^{\prime}$ is nilpotent. Hence $b$ is idempotent and is also nilpotent to $a_{0}$. This contradiction proves $a_{0}=a_{1}$. Similarly if $a_{0}>a_{1}$.

Hence $X$ only admits the trivial continuous homomorphism into a topological semigroup on the unit interval. Indeed, we see that $X$ admits no continuous homomorphism onto a one-dimensional semigroup.

Thus we have proved the following

THEOREM 2. There exists a compact, arcwise-connected, Hausdorff, abelian topological semigroup which is two-dimensional and has zero and identity but admits no continuous homomorphism onto a one-dimensional semigroup.

This includes the solution of problem P6 of Mostert and Shields.

\section{REFERENCES}

1. W. M. Faucett, Compact semigroups irreducibly connected between two idempotents, Proc. Amer. Math. Soc. 6 (1955), 741-747.

2. P. S. Mostert and A. L. Shields, On the structure of semigroups on a compact manifold with boundary, Ann. of Math. (2) 65 (1957), 117-143.

The Mathematicai, Institute, OXford 